\title{
Modeling the meteoric dust effect on the Equatorial Electrojet
}

\author{
P. Muralikrishna (1) and V.H. Kulkarni (2)
}

(1) Instituto Nacional de Pesquisas Espaciais - INPE/MCT, C.P.515, 12201-970, São José dos Campos - SP, Brazil (2) V.H.Kulkarni, 10, Archen Co-Op Housing Society, Sector - 4, Vashi, New-Bombay, 400 703, India

Dust particles of meteoric origin the lower E-region can affect the conductivity parameters by varying the effective collision frequency and by causing electron bite outs through the capture of ambient electrons. In a magnetized plasma, assuming a realistic model for the distribution of meteoric dust particles and effective collision cross section by an equivalent number of neutral dust particles, the Hall and the Pederson conductivities are obtained. The Cowling conductivity profile is calculated using model electron densities in the ionospheric E-Region and the neutral density model as given by the MSIS-E-90 for the equatorial region. The collision parameters can be considerably different from those estimated from atmospheric models, in the lower E-region heights where dust particles of meteoric origin are known to exist in large numbers. It is known that a considerable fraction of these dust particles may capture free electrons from the ambient medium and get charged negatively. This can result in reduction of the number density of free electrons especially below the electrojet peak where the dust particles can be present in large numbers, at least on days of large meteor showers. This can alter the vertical profile of the east-west Hall current driven by the vertical Hall polarization field and under favorable conditions, can even account for the reversal of the electrojet currents below the current peak. 